\title{
Fabrication of metal matrix composites under intensive shearing
}

\author{
N. Hari Babu' ${ }^{a}$ S.Tzamtzis ${ }^{b}$, N. Barekar ${ }^{c}$, J. B. Patel ${ }^{d}$ and Z. Fan ${ }^{e}$ \\ BCAST, Brunel University, Uxbridge, UB8 3PH, UK \\ amtsthbn@brunel.ac.uk, ${ }^{b}$ mepgsst@brunel.ac.uk, ${ }^{\mathrm{c} m e s r n s b @ b r u n e l . a c . u k, ~}$ \\ 'mesrjbp@brunel.ac.uk, ${ }^{\mathrm{e}} \mathrm{mtstzzf@brunel.ac.uk}$
}

Key Words: Intensive Shearing, Metal Matrix Composites, Rheo-process

\begin{abstract}
Current processing methods for metal matrix composites (MMC) often produces agglomerated reinforced particles in the ductile matrix and also form unwanted brittle secondary phases due to chemical reaction between matrix and the reinforcement. As a result they exhibit extremely low ductility. In addition to the low ductility, the current processing methods are not economical for producing engineering components. In this paper we demonstrate that these problems can be solved to a certain extent by a novel rheo-process. The key step in this process is application of sufficient shear stress on particulate clusters embedded in liquid metal to overcome the average cohesive force of the clusters. Very high shear stress can be achieved by using the specially designed twin-screw machine, developed at Brunel University, in which the liquid undergoes high shear stress and high intensity of turbulence. Experiments with $\mathrm{Al}$ alloys and $\mathrm{SiC}$ reinforcement reveal that, under high shear stress and turbulence conditions Al liquid penetrates into the clusters and disperse the individual particle within the cluster, thus leading to a uniform microstructure.
\end{abstract}

\section{Introduction}

Demands set by the automotive and aerospace industries have led to the development of innovative combinations of composite materials. A large majority of these materials are metal matrix composites (MMC) in which metallic matrices are reinforced with high strength and high modulus phases, such as carbides, nitrides, and oxides. Particulate MMCs have been shown to offer improvements in strength, wear resistance, structural efficiency, reliability and control of physical properties such as density and coefficient of thermal expansion, thereby providing improved engineering performance in comparison to the un-reinforced matrix [1-8]. Several authors [1,3,4] reviewed the applications for MMCs and major markets of ground transportation, thermal management, aerospace, industrial, recreational and infrastructures. In all these applications, the principal advantage of MMCs is that their physical and mechanical properties can be enhanced to desired level by tailoring their microstructures for specific engineering applications.

MMCs are generally processed with liquid metal routes such as stir casting and infiltration. Powder metallurgy route is also used for specific applications. However, infiltration route is most commonly used method by the industries and accounts for largest volume in primary production $(\sim 60 \%)$ [7]. In this method, a ceramic preform of the desired shape is infiltrated with the liquid metal by the application of a pressure or by pressure-less techniques. It was first widely used in the automotive industry but is now also a preferred process in the thermal management industry as a result of the ability to produce high quality near net shape or net shape components. Due to the high volume fraction of ceramic reinforcements, liquid metal infiltration is not appropriate for components that require thermo-mechanical deformation or for fracture sensitive applications. Additional process called insertion casting method has to be employed to dilute the reinforcement content to the required level.

To obtain a specific mechanical/physical property, ideally speaking, the MMC should consist of fine particulates distributed uniformly in a ductile matrix and with clean interface between particulate and matrix. However, the current processing methods often produce 
agglomerated particles in the ductile matrix and as a result they exhibit extremely low ductility $[9,10]$. Low ductility and high cost of producing components of even minimally complex shape and presence of reactive layer, due to chemical reaction, at particulate/matrix interface hinders the widespread adoption of MMCs for engineering applications. Agglomeration induces unwanted brittle nature to the MMC and is more severe when the particulate size is in sub-micron or nanoscale range. Such microstructural inhomogeneity can create strain gradients locally and reduces the tensile strength significantly [11]. Previous attempts to fragment the agglomerates in the liquid metal at microscopic scale have been unsuccessful [1-10]. Severe agglomeration nature of nanoparticles, due to high cohesive energy, combined with lack of dispersive technology for mixing nonwettable nano-particles have hindered the progress in fabrication of high performance nanoparticulate MMCs with liquid processing routes. In addition to the microstructural inhomogeneities, the current processing methods are not economical due to many processing steps. Increased processing steps at elevated temperatures aid the chemical reaction between matrix and particle, which often resulting in brittle secondary phases [12]. These problems can be addressed by developing an efficient process for reducing agglomeration and processing time in the liquid state. We refer this process as rheo-process in this paper and it is based on application of sufficient shear stress $(\tau)$ on particulate clusters embedded in liquid metal to overcome the average cohesive force or the tensile strength of the cluster. This paper presents the concept of applying intensive shearing to disagglomerate particulates and an example of processing of $\mathrm{Al} / \mathrm{SiC}$ metal matrix composites using this novel rheo-process is discussed.

\section{Experimental}

The matrix material is commercially available LM25 alloy with chemical composition of Al $8.18 \% \mathrm{Si}-0.3 \% \mathrm{Cu}-0.3 \% \mathrm{Mg}-0.16 \% \mathrm{Mn}-0.5 \% \mathrm{Fe}-0.25 \% \mathrm{Zn}-0.01 \% \mathrm{Cr}-0.05 \% \mathrm{Ti}$. Alloy was initially melted above $700{ }^{\circ} \mathrm{C}$ in graphite crucible. The ceramic reinforcement phase was SiC with average particle size $\sim 4 \mu \mathrm{m}$. $5 \mathrm{Vol} \%$ of $\mathrm{SiC}$, was pre-heated to $400{ }^{\mathrm{O}} \mathrm{C}$ and poured slowly and continuously into liquid metal that is being stirred with a steel impeller (coated with BN), at 800 $\mathrm{rpm}$. The mixing process was carried out during slow cooling between $640{ }^{\circ} \mathrm{C}$ and $600{ }^{\circ} \mathrm{C}$ (below the semi-solid temperature of this alloy). In order to improve the fluidity of the mixed slurry, its temperature was increased to approximately $630{ }^{\circ} \mathrm{C}$ and a small portion of the liquid was cast into a steel mould for the purpose of investigating microstructure. To introduce high shear rate to this slurry, the remaining pre-mixed $\mathrm{Al} / \mathrm{SiC}$ slurry was transferred to twin-screw machine which was maintained at required temperatures (e.g. $612{ }^{\circ} \mathrm{C}$ and $630{ }^{\circ} \mathrm{C}$ ). The rotation speed for twin-screws was maintained at $800 \mathrm{rpm}$ for $60 \mathrm{~S}$. Sheared slurry was transferred to a steel die and the microstructures of the solidified MMCs were investigated using optical microscopy.

\section{Results and Discussion}

Conventional MMC processing methods consist of multiple processing steps, whereas, the rheoprocess is a simple process and primarily consists of (a) distributive mixing and (b) dispersive mixing under intensive shearing prior to shaping.

(a) Distributive mixing: A stirring process with a steel impeller has been employed to pre-mix the $\mathrm{Al}$ melt with hard $\mathrm{SiC}$ particulates. It is known that the wettability of $\mathrm{SiC}$ with $\mathrm{Al}$ liquid is generally poor. Prior to optimise the pre-mixing process, several experiments were carried out to mix $\mathrm{SiC}$ at various temperatures above liquidus temperature. Based on these experiments, it has been found that it is essential to mix $\mathrm{SiC}$ particles in semi-solid state to improve the wettability and thereby to improve spatial homogeneity of particulates or particulate clusters (similar volume fraction in any part of the mixture). As the impeller rotates, at moderate speeds (up to $800 \mathrm{rpm}$ ), a vortex is generated in the melt that draws the reinforcement particles into the melt from the surface. Creation of high level of shear force is necessary for $\mathrm{Al} / \mathrm{SiC}$ mixture because of poor wetting of SiC with $\mathrm{Al}$. In a poor wetting system, the pressure required to force a liquid to enter porus agglomerate 
increases as the pore size decreases. Impeller at high rotating speed exerts a high and local shear on the particulate clusters and is especially useful in breaking down aggregates of bulk cohesive $\mathrm{SiC}$ powder. In order to produce adequate melt circulation and homogeneous distribution of the reinforcement throughout the matrix material, the impeller design plays crucial role. High turbulent flow in the axial direction was necessary to obtain fairly uniform particle distribution. The stirring condition, melt temperature, and the type, amount and nature of the particles are some of the main factors that are being investigated further $[13,14]$. The process parameters for distributive mixing are to be optimised further by conducting experiments with various types of impellers designed to achieve homogeneous distributive mixing in chemical, pharmaceutical, mining and food processing technologies [15].

Local distribution of added particulate lumps can be distributed relatively uniformly by applying shear force on the clusters. The force on a cluster is directly related to the medium viscosity. The maximum force on a particle cluster rotating in a shear flow, in the vicinity of stirrer, is given by

$$
F=6 \pi \eta a^{2} \dot{\gamma}
$$

where $a$ is the radius of each primary particle in the cluster, $\eta$ is the viscosity and $\dot{\gamma}$ is the shear rate in the surrounding liquid medium. Laminar flow, induced in the liquid by the impeller, is associated normally with high viscosity fluids. Under this type of flow inertial forces quickly die out under the action of the high viscosity. Therefore, rotating impellers must occupy a significant proportion of the crucible if adequate bulk motion is to be achieved. Close to these rotating surfaces, large velocity gradients exist. These are laminar regions of high shear rate which can cause fluid elements to be deformed and stretched. Solid agglomerates, within the high-viscosity fluid, experience high shear stresses which can lead to agglomerate break-up and homogenization. In order to distribute the $\mathrm{SiC}$ lumps into small aggregates, it is important to increase the shear force via increasing the stirrer speed. Figure 1 shows the microstructure of a solidified distributive mixed melt in a steel die. It can be seen that the 2-4 micron SiC particles (dark in contrast) are dispersed in Al alloy (white in contrast). In addition, clusters based on SiC particles are a common feature in these microstructures. Similar clustering behaviour is observed in entire volume of the samples. The degree of mixing is governed by the momentum transfer from the position of the stirrer to the clusters located away from the stirrer position. In conventional impeller used in this study, although high shear force can be applied to the liquid that is in contact with the impeller, the presence of velocity gradients within the liquid media results in low shear force when averaged out of the whole volume of the liquid media. Thus the degree of mixing is limited for the clusters located away from the impeller when mixed using conventional impellers.

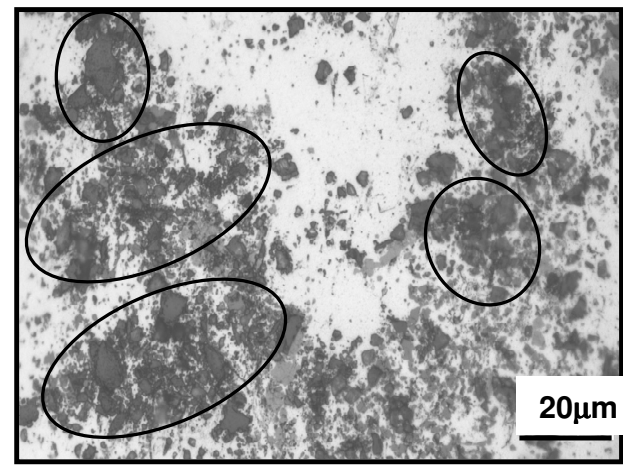

Fig. 1. Micrograph of $\mathrm{Al} / \mathrm{SiC}$ composite solidified from distributive mixed melt. Agglomerate behaviour of $\mathrm{SiC}$ particles is commonly observed.

(b) Dispersive mixing with intensive shearing: To break agglomerates into individual particles in liquid metal, the applied shear stress $(\tau)$ on particulate clusters should overcome the average 
cohesive force or the tensile strength of the cluster. High shear stress on cluster can lead to dispersion in the form of erosion (gradual shearing off). Wetting behaviour between ceramic particles and liquid metal is generally very poor. However, under high shearing and turbulence conditions, liquid can penetrate into the clusters and displace the individual particles within the cluster. The displacement distance of the particle from the cluster centre can be tuned by controlling the applied shear stress on the clusters.

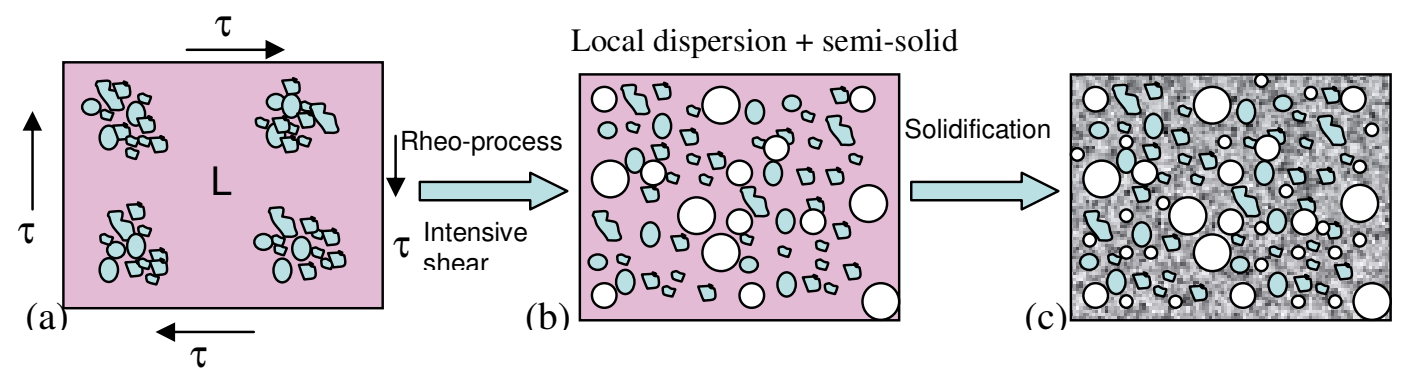

Fig.2. Schematic illustration of microstructures produced by (a) conventional stirring, liquid metal with reinforcement particles, resulting in cluster formation (b) rheo-process (intensive sheared melt with high turbulence) resulting in uniformly distributed particles at microscopic level and fine globular primary solidified metallic phase (c) cast sample from sheared melt shown in Fig. (b).

Applying high shear stress on melt (Fig. 2a) has not been tested so far due primarily to the lack of effective mixing technology. The maximum applied shear stress using conventional stirrers is around 200-400 $\mathrm{Pa}$ depending on type of stirrer and viscosity of the liquid. For Aluminium alloys, when the melt is in semi-solid state, very high shear stress (up to $50 \mathrm{MPa}$ ) can be achieved by using the specially designed twin-screw machine, developed at Brunel University, in which the liquid undergoes high shear stress and high intensity of turbulence [16,17]. The twin-screw slurry maker has a pair of co-rotating and self-wiping screws rotating inside a barrel. The screws have specially designed profiles to achieve high shear rate and high intensity of turbulence [16,17].

When liquid/particulate slurry, mixed using a conventional impeller, was poured into a twinscrew machine in which twin-screws were rotating at high speeds, the slurry undergoes a shear deformation with cyclic variation of shear rate. The shear stress $(\tau)$ is given by

$$
\tau=\eta \pi N(\mathrm{D} / \mathrm{G}-2)
$$

where $\eta$ is the viscosity, $N$ is the rotating speed of the screw, D is the outer diameter of the screw, and $G$ is the gap between screw flight and the barrel surface. Viscosity of Al alloys in semi-solid state is about $200 \mathrm{PaS}$ and at a twin-screw rotation speed of $800 \mathrm{rpm}$, using Eq. (2) the shear stress on the liquid slurry is calculated to be $35 \mathrm{MPa}$ which is well above the tensile strength $(T)$ for cohesive powder agglomerate. According to Kendall's model [18],

$$
T=11.03 \varphi^{4} \Gamma_{\mathrm{c}}^{5 / 6} \Gamma^{1 / 6} /\left(\mathrm{l}_{\mathrm{f}} \mathrm{a}\right)^{1 / 2},
$$

where $\varphi, a, \Gamma_{c}, \Gamma$ and $l_{f}$ are the volume fraction of particulates, agglomerate size, fracture surface energy and the equilibrium surface energy and the flaw/void size in the cluster, respectively. The tensile strength for highly cohesive nature of particulates was reported to be a maximum of $300 \mathrm{KPa}$ [19]. In the rheo-process, the applied shear stress $(\tau)$ with twin-screw will be maintained higher than the tensile strength of agglomerate, $T$. Unlike in distributive mixing with conventional impeller, the application of high shear stress with twin-screw results in small fragments or individual $\mathrm{SiC}$ particles as shown in Fig. 3a. Based on the assumption that agglomerates are a collection of spherical particles, Rumpf [20] calculated the tensile strength and suggests that

$$
T \alpha F_{d} d d^{2}
$$


where $F_{c}$ is inter particle cohesive force and $d$ is the diameter of the particle. The smaller the particle the higher the tensile strength and similarly the shorter the inter particle distance, which is likely the case for fine powder, the higher the tensile strength, thus needing high shear stress to be applied to break the clusters.

In this study, application of high shear stress with twin screw machine was carried out at two different temperatures, one being in semi-solid state $\left(612{ }^{\circ} \mathrm{C}\right)$ and the other being above the liquidus temperature $\left(630{ }^{\circ} \mathrm{C}\right)$. When the twin-screw barrel temperature was at $612{ }^{\circ} \mathrm{C}$, which is below the liquidus temperature $\left(615{ }^{\circ} \mathrm{C}\right.$ for LM25 system), the distributive mixed slurry will be rapidly cooled to the semi-solid processing temperature while being sheared by the rotating twinscrew converting the liquid into semisolid slurry with fine and spherical primary Al-phase particles (illustrated as white circles in Fig. 2b). These fine and globular primary matrix particles (white in contrast grains in Fig.2b\&c) in the liquid retain their shape and size during casting [21]. Fine grain structure increases simultaneously the tensile strength and the plastic behaviour of the matrix [22]. Figure 3a shows typical microstructure of $\mathrm{Al}$ composite containing $5 \mathrm{vol} \%$ of $\mathrm{SiC}$ solidified from intensively sheared melt below liquidus temperature. A great contrast in the homogeneity of SiC particulates can be seen between samples produced with conventional stir cast (Fig. 1) and with the novel rheo-process (Fig. 3a). Intensive shearing solves the most significant problem (i.e, the difficulty of achieving a homogeneous distribution of reinforcement in the matrix composites) associated with the production of MMCs. Successful dispersion of 1-5 micron sized SiC particles within the metal matrix in microscopic scale can be clearly seen in Fig. 3a. We have demonstrated that processing of MMCs containing as low as 1-5 $\mu \mathrm{m}$ sized SiC particulates without the presence of clusters, but with good distribution is now possible. The average inter-particle distance of $\sim 1-2$ $\mu \mathrm{m}$ in MMCs is now a reality in a liquid processing method. Such homogeneous distribution of reinforcement is essential for obtaining optimum mechanical properties. For MMCs processed with conventional liquid processing routes, $\mathrm{SiC}$ particulates are known to segregate between primary phase grains. This was explained by many authors [23] that the growth front of the primary Alphase pushes these particles ahead of the growth front, thus leading to a trail of $\mathrm{SiC}$ particles along the grain boundaries. Such segregation is not common, as shown in Fig. 3a, when the intensively sheared semi-solid melt is solidified. The mechanical strength of the primary phase that is nucleated just below the liquidus temperature is very poor at that temperature and hard SiC particulates can easily penetrate into these primary phases during intensive shearing. In other words, the tensile strength of the primary Al-phase is much lower than the shear stress that is applied by the twinscrew machine and hence the primary Al-phase can entrap the SiC particles. In MMCs, homogeneous microstructure is highly desirable to achieve the superior strength while retaining ductility. However, Al/SiC slurry in semi-solid state exhibits very poor fluidity and transforming this slurry into required shapes is not practical.
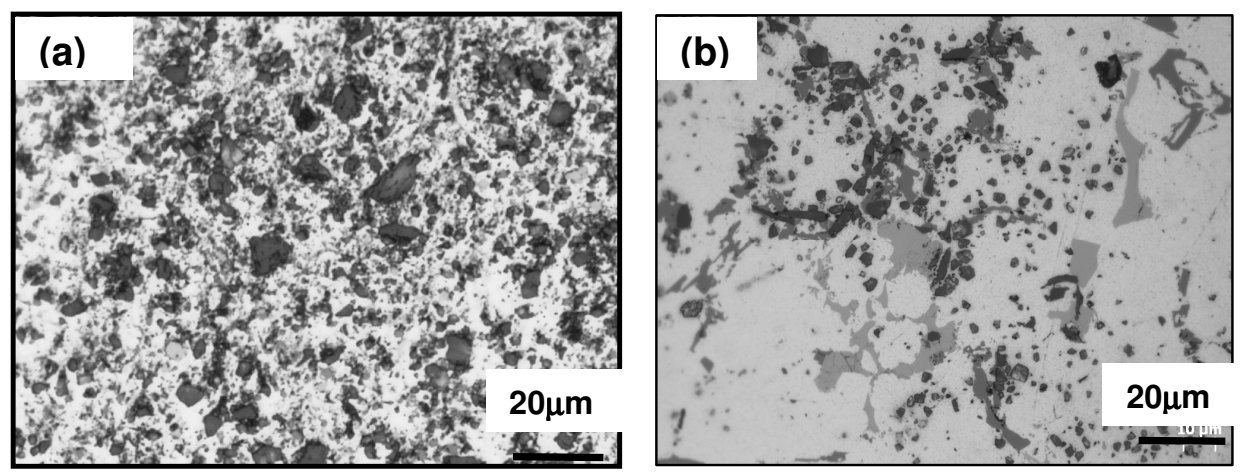

Fig 3. Optical micrographs of $\mathrm{Al} / \mathrm{SiC}$ composites solidified from the intensively sheared melt at temperatures (a) $612{ }^{\circ} \mathrm{C}$ in semi-solid temperature range and (b) at $630{ }^{\circ} \mathrm{C}$ in liquid state.

To improve the fluidity of $\mathrm{Al} / \mathrm{SiC}$ slurry and to die-cast the sheared slurry into required shapes, it is necessary to increase the shearing temperature above the liquidus temperature. Figure 
3(b) shows microstructure of solidified melt after intensively shearing the melt at $630{ }^{\circ} \mathrm{C}$ for $60 \mathrm{~S}$. Dispersion of SiC particulates can be clearly seen in this case also, however, the SiC particulates tend to segregate along the grain boundaries. In some areas of samples, entrapment of SiC particles by the primary Al-phase growth front is also observed, but the entrapment is less pronounced. Fine and homogeneous microstructure, unlike conventional MMC, rheo-processed MMC, is expected to improve simultaneously the tensile strength and ductility [24]. Simultaneous improvement in the mechanical strength and ductility has been observed in these rheoprocessed $\mathrm{Al} / \mathrm{SiC}$ composites and the detailed results will be published elsewhere $[13,14]$. High resolution microscope shows presence of clean interface between $\mathrm{Al} / \mathrm{SiC}$ matrix for most of the $\mathrm{SiC}$ particulates that are embedded in $\mathrm{Al}$ alloy matrix, suggesting that the rheo-process (mixing liquid with particulates under intensive shearing) results in absence of chemical reaction between the particle and liquid metal due primarily to the short time involved in mixing process. Absence of chemical reaction is highly desirable from the mechanical / physical property view points.

\section{Conclusion}

A new novel rheo-process has been developed to fabricate metal matrix composites with uniform microstructures and without formation of particulate agglomerates. This rheo-process is based on intensive shearing of liquid metal containing particulate clusters. Intensive shearing is observed to disperse the agglomerates into individual particle within the alloy matrix and solves the most significant problem (i.e, the difficulty of achieving a homogeneous distribution of reinforcement in the matrix composites) associated with the production of MMCs. We have demonstrated that processing of MMCs containing as low as 1-5 $\mu \mathrm{m}$ sized SiC particulates without the presence of clusters, but with good distribution using a liquid processing route, is achievable. In addition to homogeneous microstructures, overall processing steps to produce Rheo-MMC are significantly low, when compared to that of commonly employed industrial process.

\section{References}

[1] T.W. Clyne and P. J. Withers: Cambridge University Press (1993).

[2] D. J. Lloyd; Int. Mater. Rev. 39, (1994), 1

[3] P. Rohatagi: American Foundry Society Trans. (2001), 01-133

[4] S. Rawal: JOM, 53, (2001), 14

[5] N. Chawla and K. K. Chawla: JOM 58, (2006), 67

[6] T. W. Clyne and F. Simancik: Euromat 99, Wiley-VCH Verlag, 2000.

[7] http://mmc-assess.tuwien.ac.at (Data base for MMCs and their assessment for innovations)

[8] Z. Fan, P. Tsakiropoulos and A. P. Miodownik: J. Mater. Sci. 29, (1994), 141

[9] J. Segurado, C. Gonzalez and J. Llorca: Acta Materialia 51, (2003), 2355

[10] X. Deng and N. Chawla: J. Mater. Sci 41, (2006), 5731

[11] S. F. Corbin and D. S. Wilkinson: Acta Matell.Mater. 42, (1994), 1311

[12] A.J. Reeves, H. Dunlop and T. W. Clyne: Metall. Trans. 23, (1991), 970

[13] S.Tzamtzis, N. Barekar, N. Hari Babu, J. Patel and Z. Fan (To be Published)

[14] N. Barekar, S.Tzamtzis, N. H.Babu, B. K Dhindaw and Z. Fan (To be Published)

[15] Handbook of mixing technology, EKATO Ruhr- und Mischtechnik GmbH (1991).

[16] Z. Fan, M. J. Bevis and S. Ji, UK Patent, GB 2354471 A (2001).

[17] S. Ji, Z. Fan and M. J. Bevis: Mat.Sci. Eng A299 (2001), 210

[18] K. Kendall: Powder Metallurgy 31 (1988), 28

[19] Y.J Lee, D. L.Feke and I Manas-Zloczower: Chem. Eng. Sci, 48, (1993), 3363

[20] H. Rumpf: Agglomeration: 379, (Interscience Publishers, 1962)

[21] A. Das, S. Ji and Z. Fan: Acta Materialia 50, (2002), 4571

[22] Z. Fan and G. Liu: Acta Materialia 53, (2005), 4345

[23] D.M. Stefanescu, B. K. Dhindaw, S. A. Kacar, A. Moitra: Metall. Trans. A, 19 A (1988) 2847

[24] Z. Zhang and D. L. Chen: Scripta Materialia 54, (2006), 1321 